\title{
Previous Issues
}

\author{
Volume 4 Issue 1 \\ APRIL 2018 \\ ISSN: 2204-9762
}

ORIGINAL ARTICLE

\section{Building a central vascular access device registry in an adult intensive care unit: feasibility study}

Author(s): * Mari Takashima RN, BN, GradCertICUNursing, MEpi

Senior Research Assistant, Alliance for Vascular Access Teaching and Research (AVATAR) group, Menzies Health Institute Queensland, Griffith University, Nathan, Qld 4111, Australia

Email m.takashima@griffith.edu.au

Nicole C Gavin RN, BSc(Hons)Nursing, MAdvPrac

Nurse Researcher, Cancer Care Services, Royal Brisbane and Women's Hospital, Herston, QId, Australia

Alliance for Vascular Access Teaching and Research (AVATAR) group, Menzies Health Institute Queensland, School of Nursing and Midwifery, Griffith University, Nathan, Qld Australia

Emily N Larsen RN, BN, GDipHIthRes

Senior Research Assistant, Alliance for Vascular Access Teaching and Research (AVATAR) group, Menzies Health Institute Queensland, School of Nursing and Midwifery, Griffith University, Nathan, QLD, Australia

Nursing and Midwifery Research Centre, Royal Brisbane and Women's Hospital, Brisbane Qld, Australia

Sarah Northfield RN, BN, GradCertCancerNursing

Clinical Nurse (Research), Cancer Care Services, Royal Brisbane and Women's Hospital, Herston, Qld, Australia

Gabor Mihala MEng, GradCertBiostats

Alliance for Vascular Access Teaching and Research Group, Menzies Health Institute Queensland, Nathan Campus, Qld, Australia

Centre for Applied Health Economics, Menzies Health Institute Queensland, Nathan Campus, Qld, Australia

School of Medicine, Griffith University, Qld, Australia

Amanda Corley RN, BN, GradCertHSci, MAdvPrac

Nurse Researcher ICU, Critical Care Research Group

The Prince Charles Hospital and University of Queensland, Chermside, Qld, Australia

Senior Adjunct Research Fellow, Alliance for Vascular Access Teaching and Research (AVATAR) group, National Centre of Research Excellence in

Nursing Interventions, Menzies Health Institute Queensland, Griffith University, Nathan, Qld, Australia

Marc Ziegenfuss FCICM, FRCS

Director of Adult Intensive Care Services, The Prince Charles Hospital and University of Queensland, Chermside, Qld, Australia

Claire M Rickard RN, PhD

Professor, Alliance for Vascular Access Teaching and Research (AVATAR) group (Lead), Menzies Health Institute Queensland, School of Nursing and Midwifery, Griffith University, Nathan, Qld, Australia

${ }^{*}$ Corresponding author

This study is registered with ANZCTR: ACTRN12616000387426.

\section{INTRODUCTION}

Central venous access devices (CVADs), including peripherally inserted central catheters (PICCs), are required to provide vascular access for the majority of critical cart patients to deliver intravenous (IV) fluids and medications, and for haemodynamic monitoring. The goal is for CVADs to be used until the end of the required treatment, without complications that restrict/prevent device use, or trigger device removal and insertion of a replacement device. Such complications can be mechanical (for example, blockage, dislodgement, vein thrombosis, CVAD rupture), or infective (for example, local or bloodstream infections) ${ }^{1}$. Such device failures reduce patient satisfaction, prolong hospitalisation, increase health care costs and impact mortality ${ }^{2-4}$.

The incidence of CVAD failure and complications is unacceptably high. A systematic review of paediatric and neonatal CVADs reported that $25 \%$ failed before the completion of therapy ${ }^{1}$. While there is no comparable systematic review of total CVAD complications in adults, a review of central line-associated bloodstream infection (CLABSI) in the adult intensive care unit (ICU) reported a median incidence of 5.7 per 1000 catheter days ${ }^{5}$. Observational studies in adult critical care settings have reported that catheter-related bloodstream infection (CRBSI) (that is to say, CVAD is the definitive source) occurs in 2.8-3.0\%, and thrombosis in 1.0-8.4\% of CVADs 6.7 A systematic review highlighted a higher incidence of thromboembolism in PICCs in the critical care population compared to hospitalised patients on general wards $(13.9 \%, 95 \% \mathrm{Cl} 7.7-20.1 \% \text { vs } 4.9 \%, 95 \% \mathrm{Cl} 4.1-5.6 \%)^{8}$.

Clinical quality registries capture routine clinical practice outside controlled research conditions and therefore play a vital role in monitoring disease and health care delivery patterns, by providing treatment and service outcomes to clinicians, educators and policy-makers ${ }^{9}$. Recently, registries have also been used to provide comparative benchmarking of facilities, to allow improvements in health care processes, monitor adherence to clinical practice guidelines and standards, and assess the cost-effectiveness of delivered interventions ${ }^{9-13}$. Examples of such registries that currently exist are for stroke, acute myocardial syndrome, heart failure and renal failure and these have resulted in quality improvements in clinical settings ${ }^{12,14-16}$. However, existing registries capture only part of the health care system, resulting in significant gaps in knowledge about therapeutic effectiveness in many clinical areas ${ }^{17}$. Several studies have highlighted discrepancies between the clinical outcomes obtained during controlled research trial studies and those observed in routine clinical practice, confirming the need for ongoing registry monitoring ${ }^{18,19}$. Recently, a national 
arrangement for clinical quality registries was indicated and recommended by the Australian Commission on Safety and Quality in Health Care (ACSQHC) for quality improvement in health care settings ${ }^{17}$.

Despite this recommendation, the only consistently collected CVAD data are on BSI associated with haemodialysis catheters reported by the Australian Council on Healthcare Standards $(\mathrm{ACHS})^{20}$ and CLABSI reported via the Australian and New Zealand Intensive Care Society CLABSI registry

(http://www.anzics.com.au/Pages/CLABSI.aspx) ${ }^{21,22}$. There is no comparable national registry data relating to any mechanical CVAD failures in Australia. In contrast, a vast amount of CVAD data is contained at an individual hospital level within patient records; for example, electronic medical records (EMR), such as Metavision (iMDsoft Australia), are used in multiple ICUs across Australia, and potentially could be harnessed for a registry that facilitates research and quality assessment purposes ${ }^{23}$. However, the Metavision EMR used in our ICU is not yet structured to assess for specific CVAD outcomes including device failures. It is also unable to calculate CVAD days and CVAD failure per 1000 catheter days, which are important when benchmarking outcomes, both internally and externally. To become useful and accepted for registry use, a data pathway must: (1) be set up from the point of care entry (into EMR); (2) extract data to a registry; and (3) generate reports of close to real-time CVAL information and outcomes. The primary aim of this study was to examine the feasibility of establishing and maintaining a CVAD registry to measure device outcomes for adult patients with PICCs inserted in ICU.

\section{METHODS}

This study used 12 months' retrospective data (1 April 2016 to 31 March 2017) of all patients who had a PICC inserted while receiving care at a 24-bed ICU in a tertiaryreferral hospital in Australia. A list of patients with PICCs was automatically generated in Metavision by querying the 'devices' field of the EMR (see Appendix 1). The report also included PICCs that were inserted outside ICU and PICCs from previous admissions; however, these PICCs were excluded to ensure eligible PICCs were captured only once upon insertion, and not re-collected on subsequent admissions. Information was collected on this patient cohort until the following endpoints were reached: removal of PICC, patient transfer to another hospital/facility, or death. Information was gathered primarily from Metavision, and then from paper charts if patients were discharged from ICU with the PICC in situ. Baseline data (patient demographic and PICC details) and information on PICC complications and failures wert entered into the registry using the data management system Research Electronic Data Capture (REDCap, Vanderbilt University, USA). Laboratory data from the hospital electronic pathology system was accessed to obtain information regarding positive blood and /or other cultures to ascertain whether the PICC had a local infection or CLABSI. Confirmed CLABSI data was obtained from the hospital Infection Management and Prevention Service.

\section{Ethics and integrity}

The study was approved by the Royal Brisbane and Women's Hospital Human Research Ethics Committee (HREC/15/QRBW/643) and Griffith University (Ref No. 2016/058). The management and storage of data conformed to the requirements of the National Health and Medical Research Council (NHMRC) National Statement on Ethical Conduct in Human Research (2007) guidelines. No identifying participant information was recorded on the data management system, and all electronic data were de-identified.

\section{Primary outcomes}

The primary outcome was registry feasibility as defined by the ACSQHC feasibility criteria for Clinical Quality Registries ${ }^{17}$ : (i) consistency (in identifying PICC failure); (ii) governance (by meeting the requirements at the study site to establish a registry); (iii) operational requirements (sufficient resources obtained to maintain a registry); (iv) scope (capturing the entire eligible population); (v) capture of necessary data; (vi) clinically meaningful indicators (validated data collection tools); and (vii) infrastructure (ability of clinical information system to support data collection and reporting $)^{17}$.

\section{Secondary outcomes}

The secondary outcomes were: PICC utilisation ratio; PICC numbers and days (dwell-time); demographics of patients and PICC details (age, gender, weight, dwell time, primary diagnosis, CVADs in situ at time of PICC insertion, use of vesicants, insertion bundle checklist used, insertion side, insertion complications, laboratory-confirmed infection within 48 hours prior to insertion, number of lumens); reason for removal; and PICC failure per 1000 catheter days (CLABSI, occlusion, catheter-related thrombosis, migration, dislodgement, and PICC rupture).

\section{Analysis}

Statistical analysis was performed using Stata 12.1 (Stata Corp, College Station, Texas, USA). The PICC utilisation ratio was calculated by dividing the number of PICCs by the number of total patient days of all ICU patients during the data collection period ${ }^{24}$. The two-by-two contingency table was used to show the demographics of patients and PICC details by failed PICCs versus non-failed PICCs to generate hypotheses for future research. Continuous variables were expressed as mean and standard deviation. Categorical data were expressed as numbers and percentages. The incidence rate was calculated by failure/complication divided by CVAD days anc multiplying the result by $1000^{24}$.

\section{RESULTS}

\section{Feasibility outcomes}

The registry met the feasibility criteria with the exception of criteria 3 (operational requirement), criteria 5 (capturing necessary data), and criteria 7 (infrastructure). About half of all patients were transferred to a hospital ward with their PICC in situ. The clinical information system at the study site did not use an EMR for the surgical/medica wards, resulting in the research nurse hand-searching and reviewing paper medical records to obtain follow-up data post ICU-discharge. Thus, where EMRs were unavailable, the PICC removal data were difficult and time-consuming to obtain. Twenty-two per cent of PICC entries were lost to follow-up due to the unavailability of paper charts (missing or in use by other clinicians and/or researchers). As a result, we found it was not sustainable to maintain a PICC registry reliant on manual data entry without a hospital-wide EMR. However, if only used to capture insertion data, the registry successfully met six out of seven criteria, failing only criteria 3 (operational requirement) as all insertion data were available on the EMR. Moreover, the operational requirement could be met if the registry questions were built into the routine EMR data collection, entered by clinicians, and then auto-populated to the registry. See Table 1 for detailed assessment of feasibility against the ASCHQ feasibility criteria. 


\begin{tabular}{|c|c|}
\hline Criteria 1: Consistency & $\begin{array}{l}\text { - PICC failure was systematically identified in EMRs by identifying the end of PICC dwell } \\
\text { time on the device 'tab' of the EMR and searching for the 'reason for removal' on the } \\
\text { progress report. } \\
\text { - The reason for device failure was captured if it was recorded by the end users at the } \\
\text { time of cessation and if the records were locatable in case of paper records. }\end{array}$ \\
\hline Criteria 2: Governance & $\begin{array}{l}\text { - Establishment of the PICC registry received both ethics and governance approval } \\
\text { to measure patient and device outcomes in an adult intensive care population at a } \\
\text { tertiary-referral hospital. }\end{array}$ \\
\hline $\begin{array}{l}\text { Criteria 3: Operational } \\
\text { requirements }\end{array}$ & $\begin{array}{l}\text { During the 12-month pilot project, sufficient staffing and access to information } \\
\text { technology resources was obtained with the support of the Support, Explore, Excel, } \\
\text { Deliver (SEED) Innovation Funding Program. } \\
\text { - An estimation of 0.4 FTE research nurse time was allocated for this project. } \\
\text { - The research nurse required approximately 10-30mins to input data for each PICC } \\
\text { when Metavision was the data source. } \\
\text { - However, the use of a research nurse to input the data onto the registry is not } \\
\text { sustainable in the long-term future unless the EMR platform is customised to enable } \\
\text { auto-population of the registry fields. }\end{array}$ \\
\hline Criteria 4: Scope & $\begin{array}{l}\text { - The entire ICU population (225 eligible patients) was captured on this registry using } \\
\text { EMR. }\end{array}$ \\
\hline $\begin{array}{l}\text { Criteria 5: Capturing necessary } \\
\text { data }\end{array}$ & $\begin{array}{l}\text { - Patient demographics and PICC insertion and removal details were successfully } \\
\text { captured from routine fields in the EMR. } \\
\text { - Approximately half of all patients were transferred to a hospital ward with their PICC } \\
\text { in situ. } \\
\text { - The clinical information system at the study site lacked the EMR system for the } \\
\text { surgical/medical wards, therefore the PICC removal information was difficult and } \\
\text { time-consuming to obtain from the paper chart. This missing data influenced the } \\
\text { total failure proportion, CVAD failure rate and potentially underestimated CVAD days. }\end{array}$ \\
\hline $\begin{array}{l}\text { Criteria 6: Clinically meaningful } \\
\text { indicators }\end{array}$ & $\begin{array}{l}\text { - We used outcome indicators used for infection surveillance and other well } \\
\text { established CVAD failure outcomes }{ }^{1,8,25,40}\end{array}$ \\
\hline Criteria 7: Infrastructure & $\begin{array}{l}\text { - As explained for criteria 5, the study site did not have EMR in the general wards, } \\
\text { resulting in the need for a research assistant to review paper charts to collect follow- } \\
\text { up data post-ICU discharge. } \\
\text { - Although the registry was able to capture } 100 \% \text { of eligible patients from EMRs, about } \\
\text { one-fifth of these patients were lost to follow-up, due to missing data in the paper } \\
\text { charts. }\end{array}$ \\
\hline
\end{tabular}

\section{PICC utilisation ratio, catheter numbers and days}

The total length of stay of all ICU patient was 6,424 days from 1,820 ICU admissions during the study period. There were 280 PICCs inserted in 225 patients that accounted for a total of 3,000 catheter days. The PICC utilisation ratio was 0.467 . All PICCs $(100 \%)$ were inserted by medical staff using ultrasound guidance for vessel imaging and chest $x$-ray to confirm tip position. Only one PICC $(<1 \%)$ was inserted using a guidewire exchange.

\section{Demographics of patients requiring PICC, and PICC details}

A summary of the study population demographics and PICC details are listed in Table 2. Multiple indications for use could be selected for each PICC on the registry. PICCs were clinically indicated for the type of IV treatment (99\%), which included vesicants (91\%) and prolonged treatment (99\%). Triple lumen PICCs were most frequently used as per clinician preference (88.9\%).

Table 2: Study population and PICC demographics by failure ( $n=280$ PICCs) 


\begin{tabular}{|c|c|c|c|}
\hline & $\begin{array}{l}\text { Non-failed } \\
n=199\end{array}$ & $\begin{array}{l}\text { Failed } \\
n=81\end{array}$ & $\begin{array}{l}\text { Total } \\
n=\mathbf{2 8 0}\end{array}$ \\
\hline Age (years) a & $199 / 59.0(17.1)$ & $81 / 62.1$ (14.9) & 59.9 (16.5) \\
\hline Weight $(\mathrm{kg})^{\text {acd }}$ & $196 / 84.2(24.6)$ & $80 / 93.4(26.7)$ & $86.8(25.5)$ \\
\hline Dwell time (days) a & $199 / 10.0(13.1)$ & $81 / 12.5(9.7)$ & $10.7(12.3)$ \\
\hline Gender: male & $125(63)$ & $49(60)$ & $174(62)$ \\
\hline \multicolumn{4}{|l|}{ Primary diagnosis: } \\
\hline Medical & $117(59)$ & $44(54)$ & $161(58)$ \\
\hline Surgical & $82(41)$ & $37(46)$ & $119(42)$ \\
\hline Previous organ transplant & $32(16)$ & $9(11)$ & $41(15)$ \\
\hline \multicolumn{4}{|l|}{ CVADs in situ at insertion: } \\
\hline None & $75(38)$ & $20(25)$ & $95(34)$ \\
\hline One & $98(49)$ & $42(52)$ & $140(50)$ \\
\hline Two & $25(13)$ & $19(23)$ & $44(16)$ \\
\hline \multicolumn{4}{|l|}{ PICC indication: } \\
\hline Poor access & $1(0)$ & $0(0)$ & $1(0)$ \\
\hline $\begin{array}{l}\text { Choice of IV therapy } \\
\text { (prolonged/ vesicant infusion) }\end{array}$ & $197(99)$ & $81(100)$ & $278(99)$ \\
\hline Failed previous CVAD & $1(0)$ & $0(0)$ & $1(0)$ \\
\hline \multicolumn{4}{|l|}{ Vesicant use: } \\
\hline Antibiotics & $122(61)$ & $66(82)$ & $188(67)$ \\
\hline Immunosuppressants & $26(13)$ & $10(12)$ & $36(13)$ \\
\hline Parenteral nutrition & $4(2)$ & $1(1)$ & $5(2)$ \\
\hline Vasopressor & $114(57)$ & $48(59)$ & $162(58)$ \\
\hline Electrolyte & $121(61)$ & $45(56)$ & $166(59)$ \\
\hline Other & $131(66)$ & $50(62)$ & $181(65)$ \\
\hline $\begin{array}{l}\text { Insertion bundle checklist used } \\
\text { (fully or partially completed) }\end{array}$ & $65(33)$ & $32(40)$ & $97(35)$ \\
\hline Insertion side: right & $127(64)$ & $48(59)$ & $175(62)$ \\
\hline \multicolumn{4}{|l|}{ Vein used: } \\
\hline Brachial & $130(65)$ & $52(64)$ & $182(65)$ \\
\hline Other (basilic, brachiocephalic, cephalic) & $69(35)$ & $29(36)$ & $98(35)$ \\
\hline Insertion complication ${ }^{c}$ & $22(11)$ & $9(11)$ & $31(11)$ \\
\hline $\begin{array}{l}\text { Any laboratory-confirmed infection in } 48 \\
\text { hours prior to device insertion }\end{array}$ & $70(35)$ & $34(42)$ & $104(37)$ \\
\hline \multicolumn{4}{|l|}{ Number of lumens ${ }^{c}$ : } \\
\hline Two & $27(23)$ & $13(20)$ & $40(22)$ \\
\hline Three & $90(77)$ & $51(80)$ & $141(78)$ \\
\hline
\end{tabular}

Frequencies and column percentages shown: ${ }^{a}$ frequency/mean (SD) shown; $S D=$ standard deviation; ${ }^{b}$ insertion complication:

difficulties or complications occurred during or within 24 hours after device insertion; ${ }^{c}$ missing data due to incomplete patient records; ${ }^{d}$ weights were only recorded if the patient had been weighed 1 month prior to PICC insertion.

\section{PICC failures}

The primary reasons for PICC removal are listed in Table 3. There were 81 premature PICC removals (29\%) due to complications. There were four insertion failures (placement failure), 22 mechanical failures (accidental dislodgement, catheter migration, catheter rupture/fracture, catheter-related thrombosis, and occlusion) and four infection-related failures (one confirmed CLABSI by the Infection Management Service and three local infections) and 51 other premature removals (suspected CLABSI, unknown and other) (Table 3). There were 33 PICCs removed due to suspected CLABSI (12\%) despite only one CLABSI confirmed by the Infection Management and Prevention Service using the national surveillance definition ${ }^{25}$. The incidence rate of all failures ( $\mathrm{n}=81$ ) was 27.0 per 1000 catheter days $(95 \% \mathrm{CI} 21.7-33.6)$, or 16.0 failures per 1000 catheter days $(95 \% \mathrm{Cl} 12.1-21.2)$ without suspected CLABSI. (Refer to Table 3 for incident rates for individual failures.)

Table 3: Reasons for catheter removal ( $n=280)$ 


\begin{tabular}{|c|c|c|c|}
\hline & $\mathrm{N}$ & $\%$ & Incident rate $(95 \% \mathrm{Cl})$ \\
\hline \multicolumn{4}{|l|}{ No complications } \\
\hline Completed therapy & 92 & 33 & n.c. (not calculated) \\
\hline Discharged from ICU (lost to follow-up) & 61 & 22 & n.c. \\
\hline Transferred to other facility (lost to follow-up) & 24 & 9 & n.c. \\
\hline Patient died & 21 & 8 & n.c. \\
\hline Routine re-site & 1 & 0 & n.c. \\
\hline \multicolumn{4}{|l|}{ Complications } \\
\hline Suspected CLABSI & 33 & 12 & $11.0(7.82-15.5)$ \\
\hline Other* & 14 & 5 & $4.67(2.76-7.88)$ \\
\hline Occlusion+ & 7 & 3 & $2.33(1.11-4.89)$ \\
\hline Catheter-related thrombosis+ & 6 & 2 & $2.00(0.89-4.45)$ \\
\hline Accidental dislodgement+ & 5 & 2 & $1.67(0.69-4.00)$ \\
\hline Placement failure & 4 & 1 & $1.33(0.50-3.55)$ \\
\hline Unknown & 4 & 1 & $1.33(0.50-3.55)$ \\
\hline Catheter migration+ & 3 & 1 & $1.00(0.32-3.10)$ \\
\hline Local infection** & 3 & 1 & $1.00(0.32-3.10)$ \\
\hline Catheter rupture/fracture+ & 1 & 0 & $0.33(0.05-2.37)$ \\
\hline Confirmed CLABSI** & 1 & 0 & $0.33(0.05-2.37)$ \\
\hline One or more complications & 81 & 29 & n.c. \\
\hline Total & 280 & 100 & - \\
\hline
\end{tabular}

* 'Other' included: doctor's request to minimise access $(n=2)$; excess oozing from PICC site $(n=2)$; replaced with other vascular device with extra access due to patient deterioration $(n=5)$; PICC removal prior to transition/transfer $(n=5)$

+ mechanical failure

${ }^{* *}$ infective failure 


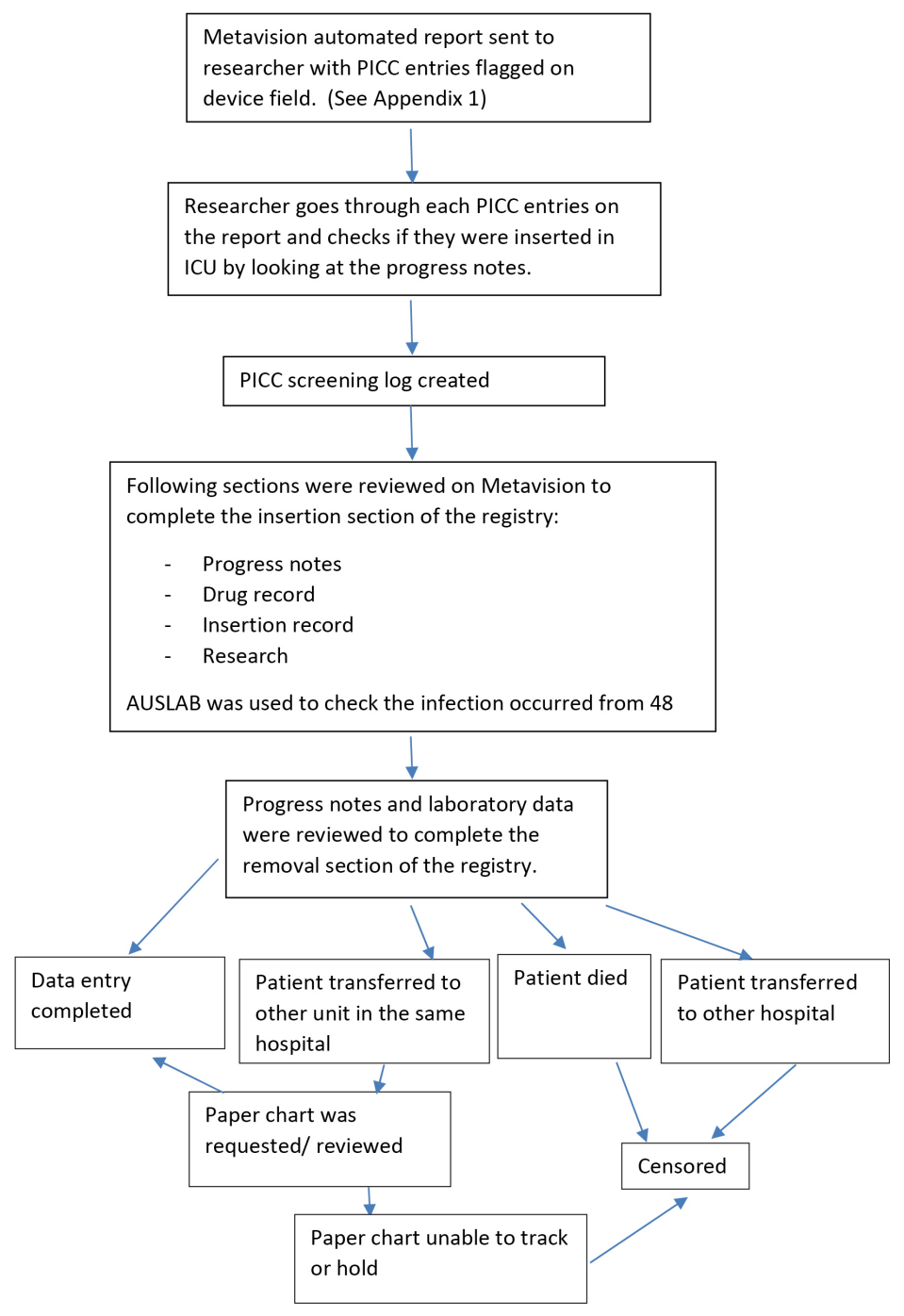

Figure 1: Flow Diagram: The process of data collection into the CVAD registry

PICC: peripherally inserted central catheter, ICU: intensive care unit.

\section{DISCUSSION}

This registry successfully collected information about patients requiring PICCs, including indication for PICCs and characteristics of PICC, when using ICU information available on the EMR, and with minimal additional time to add CLABSI outcomes from the routine data collected by the hospital. However, the registry was unable to collect information on complications and reasons for removal for the sub-group that switched from the EMR to paper charts upon ICU discharge. Insufficient information technology infrastructure resulted in $22 \%$ of data being lost to follow-up. The CLABSI rate ( 0.33 per 1000 days) was lower than a previous meta-analysis of PICC-relatec CLABSI (1.0-2.1 per 1000 catheter days) ${ }^{26}$ and slightly lower than previously published registry data (0.44/1,000 days) from 2015-201622. A previous pilot RCT conducted at the same facility reported no CLABSI or local infection ${ }^{27}$, which supports this low CLABSI rate. It was also revealed that 33 PICCs were removed due to suspected catheter infection compared with only one confirmed CLABSI, suggesting that a significant number of PICCs were removed unnecessarily or that diagnostic approaches were not adequate. This is similar to previous studies ${ }^{28,29}$; for example, in the prospective cohort study by Parienti et al. ${ }^{29}$, of 2532 catheters studied, 310 were removed for suspected CLABSI but only 26 were confirmed as catheter-related infection. Previous studies have investigated the effects of immediate, deferred or no removal of CVADs suspected of infection and found no difference in morbidity or mortality between groups $\mathrm{s}^{30,31}$. The current clinical practice guidelines for the diagnosis and management of intravascular catheter-related infection recommends that long-term catheters should be removed from patients with CRBSI with any conditions such as severe sepsis, suppurative thrombophlebitis, endocarditis, BSI that persists for longer than 72 hours despite antimicrobial therapy, or infection due to Staphylococcus aureus, Pseudomonas aeruginosa, fungi, or mycobacteria ${ }^{32}$. It is also recommended that patients with CRBSI originating from a short-term catheter (as a result of a gram-negative bacilli, $S$. aureus, enterococci, fungi, and mycobacteria) should have their device immediately removed ${ }^{32}$. However, it is questionable if this guideline is also applicable for ICU patients who are often in an unstable condition. A clear guideline for the management of CVADs suspected of infection for ICU patients is urgently needed to prevent unnecessary removal of CVADs and the associated increased risks to patients and costs to the health care system.

In a recent review of risk factors for PICC-related complications, the authors listed patient, device, and provider-associated risk factors for PICC-related BSI and venous thromboembolsim (VTE) ${ }^{26}$. Although our registry collected information on a majority of these risk factors (for example, number of prior CVADs, number of PICC lumens, dwell time, monitoring insertion-related complications, infusion of vesicants), we were not able to collect all risk factors found by the review to be significant ${ }^{26}$. The risk factors for non-cancer patients that were unavailable (as they are currently not routinely recored in our EMR) were 1) operative procedure lasting >1 hour; 2) history of VTE; and 3) PICC diameter/gauge ${ }^{26}$. However, if auto-population of registry variables can be implemented, these could be included in the future registry.

The CVAD registry met the following feasibility criteria: consistency, governance, scope, and clinical meaningful indicators. However, the registry did not meet the criteria for operational requirements, capturing necessary data, and infrastructure. This was due to the difficulty and significant time consumption of locating paper charts after many patients were discharged from the ICU with their PICC. However, it is anticipated that paper charts will eventually be replaced by hospital-wide EMRs due to technology advances in health care settings, thereby rectifying the problem with infrastructure ${ }^{33}$. Additional registry limitations were related to the use of Metavison, which did not contain some of the registry questions as data entry fields (that is to say, PICC inserted on dominant-hand side). Metavision is not yet structured to export 
data specifically for CVAD outcomes including CVAD failure, nor to calculate the incidence in CVAD days. This could be solved by adding specific data entry fields to the Metavision platform, enabling registry questions to auto-populate, thus eliminating inefficient manual data entry. Additionally, some Metavision data may have been inaccurate or inconsistent depending on the end users' assessment skills, which requires education on accurate assessment and reporting and resource allocation for such education. However, as registries reflect institutional level performance, such inaccuracies may have little impact on overall findings. Furthermore, Metavision did not allow for correct calculation of CVAD days for patients who had the same PICC over multiple ICU admissions: a problem also encountered in previous studies ${ }^{34,35}$ We propose that this limitation may be solved by allocating a unique PICC device number at time of insertion, in order to connect the PICC data between the first and subsequent admissions. However, despite inherent shortcomings, EMRs have been shown to achieve a higher degree of accuracy than manual data collection and also aid in reducing health care-associated infection ${ }^{36-38}$. For future registry projects to be successful, there is a need for infrastructure of accessible EMRs throughout the episode of care, which would preserve valuable personnel resources ${ }^{36,39}$.

This study highlighted the inefficiencies of using paper-based charts for registry implementation, as many paper charts were difficult to locate and, in some cases, never found. The EMR was superior for efficient data collection purposes as it could be viewed in multiple locations with instant access. There is a potential to involve multiple hospitals using the same EMR platform to incorporate the registry question fields, thus ensuring ongoing registry participation and inter-institutional, meaningful benchmarking.

The limitations of this registry include: no fields to enter each ward's admission and discharge date and time to specify the location and duration of where the failure and complication occurred; and the loss to follow-up of nearly one-third of patients discharged from the ICU to the ward. The limitations of this study are that it was conducted in only one hospital using one EMR system and the results may not be generalisable to other settings and platforms. In addition, we did not undertake formal timings of data collection workloads. Future registry priorities should include: incorporation of additional fields to enter the admission and discharge date from each ward restructuring of Metavision to auto-populate the registry fields at the point-of-care; and automated reporting of CVAD outcomes such as dwell time, complication and failure rates at the point-of-care. Future use of such registries will provide a valuable archive for clinicians and researchers with an interest in improving CVAD outcomes $^{2}$.

\section{CONCLUSION}

In conclusion, this study demonstrated the feasibility of establishing and maintaining a CVAD registry but only in conjunction with hospital-wide EMRs. The adoption of registry fields into the EMR platform will have greater potential for time and cost efficiency by reducing manual data entry and auto-populating the registry at point-ofcare. The increased use of EMRs across different health care settings will promote the development and subsequent increased use of registries. With the increasing emergence of clinical registries in diverse range of fields, more health care stakeholders and clinicians realise the impacts and benefits of registries on patient outcomes and health care costs.

\section{REFERENCES}

1. Ullman AJ, Marsh N, Mihala G, Cooke M \& Rickard CM. Complications of central venous access devices: A systematic review. Pediatrics 2015; 136(5):e1331-44

2. To KB \& Napolitano LM. Common complications in the critically ill patient. Surg Clin North Am 2012; 92(6):1519-57.

3. Gorski L, Hadaway L, Hagle M, McGoldrick M, Orr M \& Doellman D. Infusion therapy standards of practice. J Infus Nurs 2016; 39(Suppl 1):S1-S159.

4. Loveday HP, Wilson JA, Pratt RJ, Golsorkhi M, Tingle A, Bak A et al. epic3: national evidence-based guidelines for preventing healthcare-associated infections in NHS hospitals in England. J Hosp Infect 2014; 86(Suppl 1):S1-70.

5. Ista E, van der Hoven B, Kornelisse RF, van der Starre C, Vos MC, Boersma E et al. Effectiveness of insertion and maintenance bundles to prevent central lineassociated bloodstream infections in critically ill patients of all ages: A systematic review and meta-analysis. Lancet Infect Dis 2016; 16(6):724-34.

6. Nolan ME, Yadav H, Cawcutt KA \& Cartin-Ceba R. Complication rates among peripherally inserted central venous catheters and centrally inserted central catheters in the medical intensive care unit. J Crit Care 2016; 31(1):238-42.

7. Wilson TJ, Stetler WR \& Fletcher JJ. Comparison of catheter-related large vein thrombosis in centrally inserted versus peripherally inserted central venous lines in the neurological intensive care unit. Clin Neurol Neurosurg 2013; 115(7):879-82.

8. Chopra V, Anand S, Hickner A, Buist M, Rogers MA, Saint S et al. Risk of venous thromboembolism associated with peripherally inserted central catheters: A systematic review and meta-analysis. Lancet 2013; 382(9889):311-25.

9. Hoque DME, Kumari V, Hoque M, Ruseckaite R, Romero L \& Evans SM. Impact of clinical registries on quality of patient care and clinical outcomes: A systematic review. PLoS One 2017; 12(9):e0183667

10. McNeil JJ, Evans SM, Johnson NP \& Cameron PA. Clinical-quality registries: Their role in quality improvement. Med J Aust 2010; 192(5):244-5.

11. Schwamm LH, Fonarow GC, Reeves MJ, Pan W, Frankel MR, Smith EE et al. Get With the Guidelines-Stroke is associated with sustained improvement in care for patients hospitalized with acute stroke or transient ischemic attack. Circulation 2009; 119(1):107-15.

12. Fonarow GC, Albert NM, Curtis AB, Stough WG, Gheorghiade M, Heywood JT et al. Improving evidence-based care for heart failure in outpatient cardiology practices: primary results of the registry to improve the use of evidence-based heart failure therapies in the outpatient setting (IMPROVE HF). Circulation 2010; 122(6):585-96.

13. Girgenti C \& Moureau NL. The need for comparative data in vascular access: The rationale and design of the PICC registry. J Vasc Access 2013; 18(4):219-24.

14. Peterson ED, Roe MT, Mulgund J, DeLong ER, Lytle BL, Brindis RG et al. Association between hospital process performance and outcomes among patients with acute coronary syndromes. JAMA 2006; 295(16):1912-20.

15. Lim TO, Goh A, Lim YN \& Morad Z. Use of renal registry data for research, health-care planning and quality improvement: What can we learn from registry data it the Asia-Pacific region? Nephrology 2008; 13(8):745-52.

16. Centers for Disease Control and Prevention (CDC). Use of a registry to improve acute stroke care-seven states, 2005-2009. MMWR 2011; 60(7):206-10.

17. Australian Commission on Safety and Quality in Health Care. Framework for Australian clinical quality registries. NSW, Australia: ACSQHC, 2014. Available at: https://www.safetyandquality.gov.au/wp-content/uploads/2014/09/Framework-for-Australian-Clinical-Quality-Registries.pdf. Retrieved 19 January 2018.

18. Maclntyre K, Capewell S, Stewart S, Chalmers JW, Boyd J, Finlayson A et al. Evidence of improving prognosis in heart failure: Trends in case fatality in 66,547 patients hospitalized between 1986 and 1995. Circulation 2000; 102(10):1126-31.

19. Wennberg DE, Lucas FL, Birkmeyer JD, Bredenberg CE \& Fisher ES. Variation in carotid endarterectomy mortality in the Medicare population: Trial hospitals, volume, and patient characteristics. JAMA 1998; 279(16):1278-81.

20. Australian Council on Healthcare Standards (ACHS). Australasian Clinical Indicator Report: 2008-2015. NSW, Australia; 2016.Available at: https://www.achs.org.au/programs-services/clinical-indicator-program/australasian-clinical-indicator-report. Retrieved 19 January 2018.

21. ANZICS Centre for Outcome and Resource Evaluation. Central Line Associated Blood Stream Infection (CLABSI) prevention. Victoria, Australia: ANZICS, 2014. Available at: http://www.anzics.com.au/Pages/CLABSI.aspx. Retrieved 19 January 2018

22. ANZICS Centre for Outcome and Resource Evaluation. Central Line Associated Bloodstream Infection (CLABSI) report 2015/16. Victoria, Australia: ANZICS, 2016. Available at: http://www.anzics.com.au/Downloads/CLABSI\%20Report\%202015-16.pdf. Retrieved 19 January 2018.

23. Gliklich RE, Dreyer NA, Leavy MB, editors. Registries for evaluating patient outcomes: A user's guide. 3rd ed. Rockville, MD: Agency for Healthcare Research and Quality (US), 2014. In 15, Interfacing registries with electonic health records. Available at: https://www.ncbi.nlm.nih.gov/books/NBK208625/. Retrieved 18 January 2018

24. Centers for Disease Control and Prevention. bloodstream infection event (central line-associated bloodstream infection and non-central line-associated bloodstream infection). Device-associated module BSI. Atlanta, GA: CDC, 2017. Available at: https://www.cdc.gov/nhsn/pdfs/pscmanual/4psc_clabscurrent.pdf. Retrieved 18 January 2018.

25. Australian Commission on Safety and Quality in Health Care. Implementation guide for surveillance of central line-associated bloodstream infection. Sydney: ACSQHC; 2015. Available at: https://www.safetyandquality.gov.au/wp-content/uploads/2016/04/Implementation-Guide-for-Surveillance-of-Central-LineAssociated-Bloodstream-Infection-2016-Edition.pdf. Retrieved 19 January 2018.

26. Chopra V, Anand S, Krein SL, Chenoweth C \& Saint S. Bloodstream infection, venous thrombosis, and peripherally inserted central catheters: Reappraising the evidence. Am J Med 2012; 125(8):733-41.

27. Rickard CM, Edwards M, Spooner AJ, Mihala G, Marsh N, Best J et al. A 4-arm randomized controlled pilot trial of innovative solutions for jugular central venous access device securement in 221 cardiac surgical patients. J Crit Care 2016; 36:35-42.

28. Arvaniti K, Lathyris D, Clouva-Molyvdas P, Haidich AB, Mouloudi E, Synnefaki E et al. Comparison of Oligon catheters and chlorhexidine-impregnated sponges with standard multilumen central venous catheters for prevention of associated colonization and infections in intensive care unit patients: A multicenter, randomized, controlled study. Crit Care 2012; 40(2):420-9. 
29. Parienti JJ, Mongardon N, Mégarbane B, Mira JP, Kalfon P, Gros A et al. Intravascular complications of central venous catheterization by insertion site. N Engl J Med 2015; 373(13):1220-9.

30. Lorente L, Martin MM, Vidal P, Rebollo S, Ostabal MI \& Sole-Violan J. Should central venous catheter be systematically removed in patients with suspected catheter related infection? Crit Care 2014; 18(5):564

31. Rijnders BJ, Peetermans WE, Verwaest C, Wilmer A, \& Van Wijngaerden E. Watchful waiting versus immediate catheter removal in ICU patients with suspected catheter-related infection: a randomized trial. Intensive Care Med 2004; 30(6):1073-80.

32. Mermel LA, Allon M, Bouza E, Craven DE, Flynn P, O'Grady NP et al. Clinical practice guidelines for the diagnosis and management of intravascular catheterrelated infection: 2009 update by the Infectious Diseases Society of America. Clin Infect Dis 2009; 49(1):1-45.

33. Australian Government Department of Health. The impact of the implementation on electronic ordering on hospital pathology services. Canberra: Department of Health; 2013. Available at http://www.health.gov.au/internet/publications/publishing.nsf/Content/QUPP-Electronic-Pathology-Ordering-in-Hospitals section-IIIIntroduction the-electronic-medical-record. Retrieved 19 January 2018.

34. Ippolito P, Larson EL, Furuya EY, Liu J \& Seres DS. Utility of electronic medical records to assess the relationship between parenteral nutrition and central lineassociated bloodstream infections in adult hospitalized patients. J Parenter Enteral Nutr 2015; 39(8):929-34.

35. Tejedor SC, Garrett G, Jacob JT, Meyer E, Reyes MD, Robichaux C et al. Electronic documentation of central venous catheter-days: Validation is essential. Infect Control Hosp Epidemiol 2013; 34(9):900-7.

36. Wright M, Fisher A, John M, Reynolds K, Peterson LR \& Robicsek A. The electronic medical record as a tool for infection surveillance: Successful automation of device-days. Am J Infect Control 2009; 37(5):364-70.

37. Hermon A, Pain T, Beckett P, Jerrett $\mathrm{H}$, Llewellyn N, Lawrence P et al. Improving compliance with central venous catheter care bundles using electronic records. Nurs Crit Care 2015; 20(4):196-203.

38. Allen GB, Miller V, Nicholas C, Hess S, Cordes MK, Fortune JB et al. A multitiered strategy of simulation training, kit consolidation, and electronic documentation is associated with a reduction in central line-associated bloodstream infections. Am J Infect Control 2014; 42(6):643-8.

39. Soderberg LM. Designing and creating a central venous catheter database and electronic charting system. J Intraven Nurs 2001;24(3):159-68.

40. Chopra V, O'Horo JC, Rogers MA, Maki DG \& Safdar N. The risk of bloodstream infection associated with peripherally inserted central catheters compared with central venous catheters in adults: A systematic review and meta-analysis. Infect Control Hosp Epidemiol 2013; 34(9):908-18.

\section{Appendix 1}
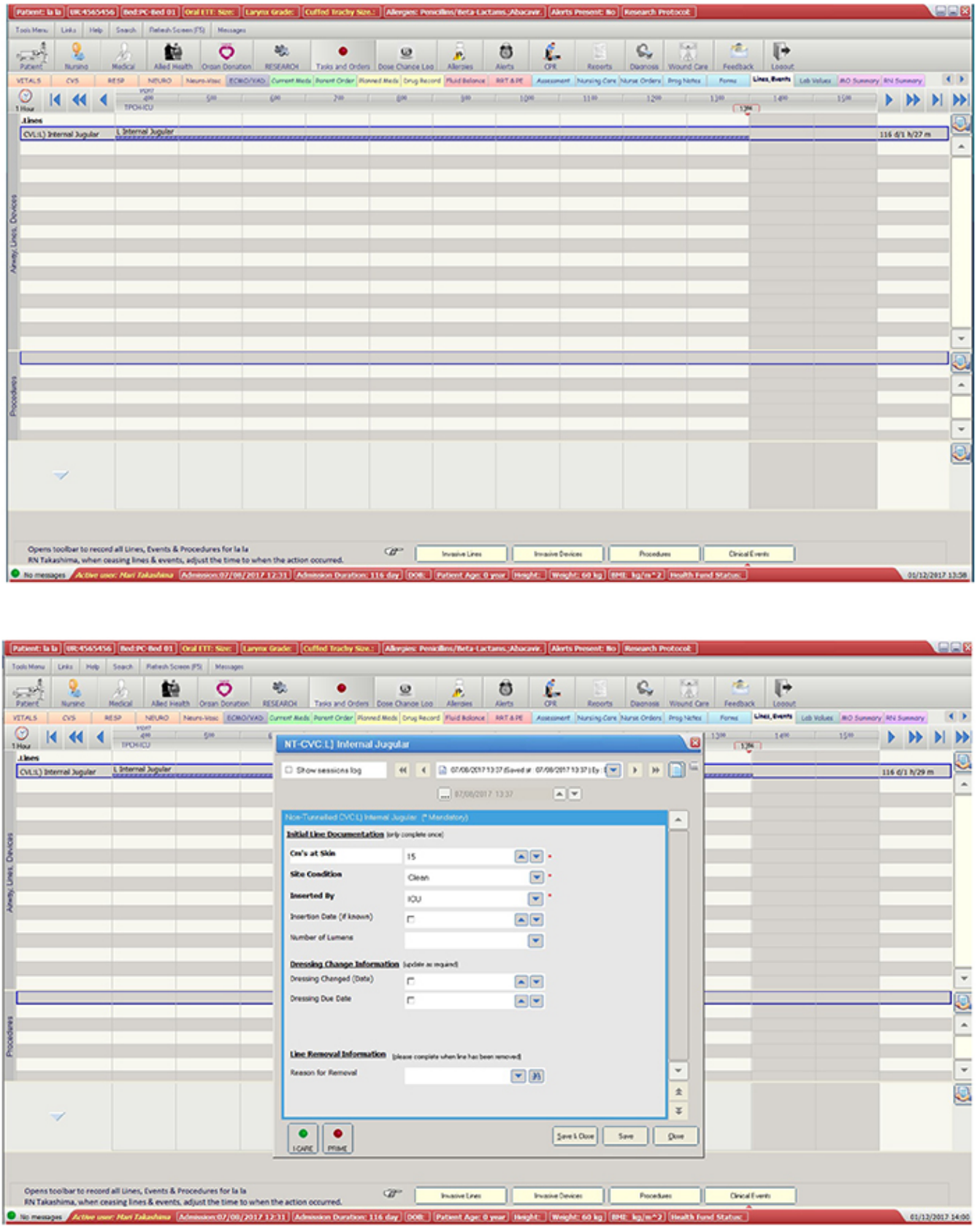


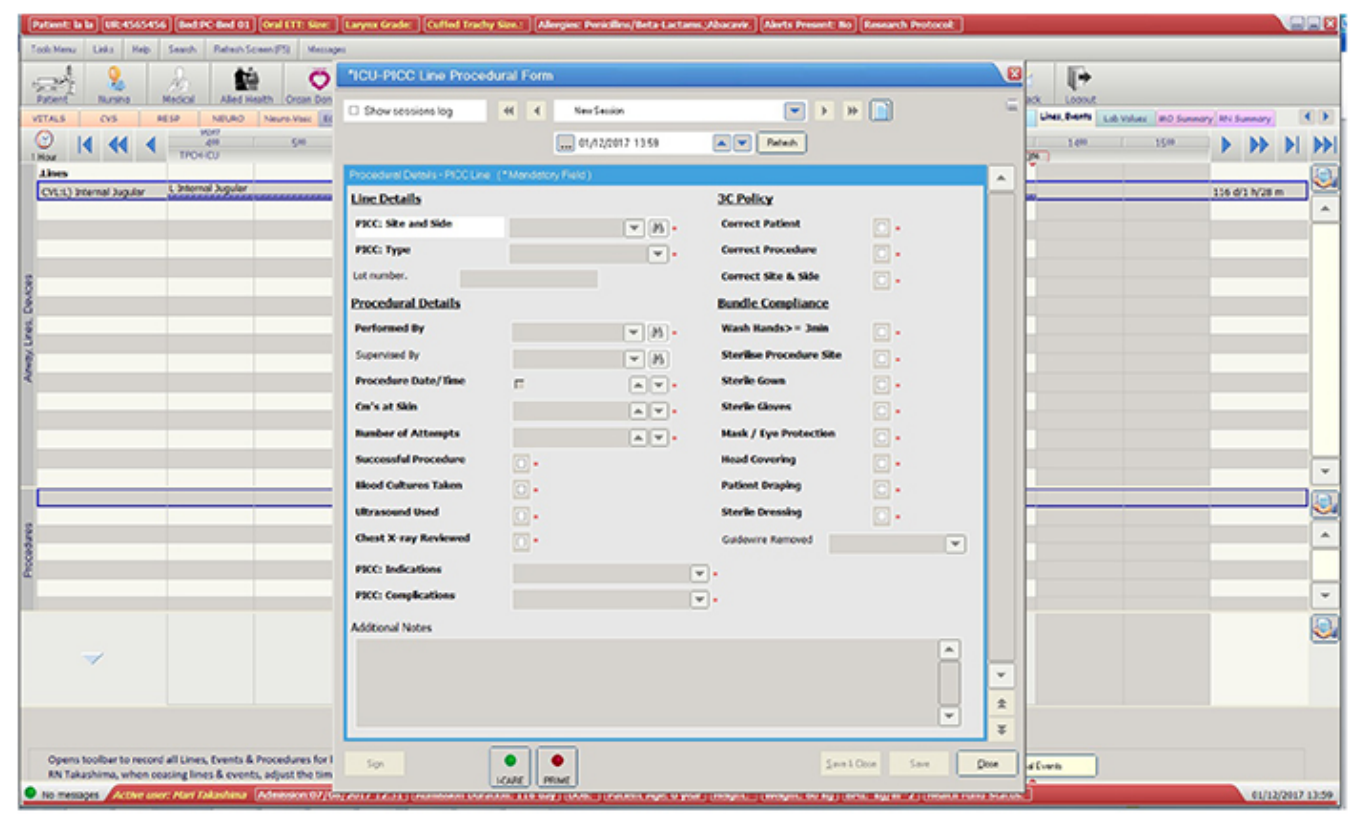

